\title{
On our bookshelf
}

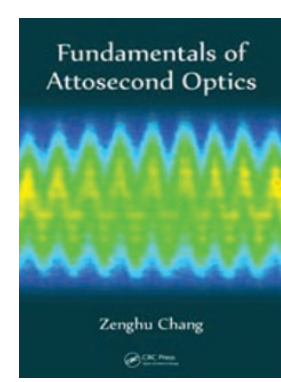

\section{Fundamentals of Attosecond Optics \\ by Zenghu Chang}

CRC PRESS. 547PP.

$\$ 89.95$

ISBN: 9781420089370

Attosecond optical pulse generation, together with the related process of highorder harmonic generation, is redefining ultrafast physics and chemistry. This book provides a focused introduction that covers the underlying concepts and techniques of attosecond optical pulse generation, as well as recent research advances that are driving the field forwards. It begins with basic theory and gradually advances onto more complex ideas. Using both semiclassical models and quantum mechanical theories, the book explains foundational concepts and mechanisms such as femtosecond lasers, high-order harmonic generation and the technological leap that inspired attosecond pulse generation. The book also introduces techniques for generating attosecond pulse trains using the basis of high-order harmonics, followed by an explanation of gating methods for extracting single isolated pulses.

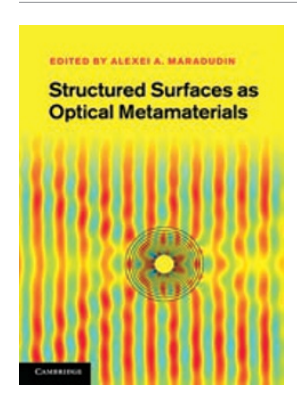

\section{Structured Surfaces as Optical Metamaterials \\ by Alexei A. Maradudin \\ CAMBRIDGE UNIVERS- ITY PRESS. 460PP. $\$ 115.00$ ISBN: 9780521119610}

This book gives an overview of a rapidly developing class of optical metamaterials that can be manipulated by suitably structuring the surfaces with which they interact. Such metamaterials have applications in a variety of fields, including materials science, photovoltaic technology, imaging and lensing, beam shaping and lasing. This publication is aimed not only at researchers and professionals working in metamaterials and plasmonics, but also at those just entering this exciting new field. The author discusses the range of different structured surfaces, their design, fabrication and unusual optical properties, recent experimental observations and potential applications.

\begin{tabular}{|l|l}
\hline C) & $\begin{array}{l}\text { Twisted Photons } \\
\text { by Juan P. Torres and } \\
\text { Lluis Torner }\end{array}$ \\
WILEY. 288PP. \$195.00 \\
ISBN: 9783527409075
\end{tabular}

In most practical scenarios, the angular momentum of light can be decomposed into two independent contributions: spin angular momentum and orbital angular momentum. The orbital contribution contributes a fundamentally new degree of freedom to light, with fascinating and widespread applications. Unlike spin angular momentum, which is associated with the polarization of light, orbital angular momentum arises as a consequence of the spatial distribution of the intensity and phase of an optical field, even down to the single-photon limit. The authors deal with how orbital angular momentum can impact our understanding of the ways in which light and matter interact, as well as its practical potential throughout different areas of science and technology.

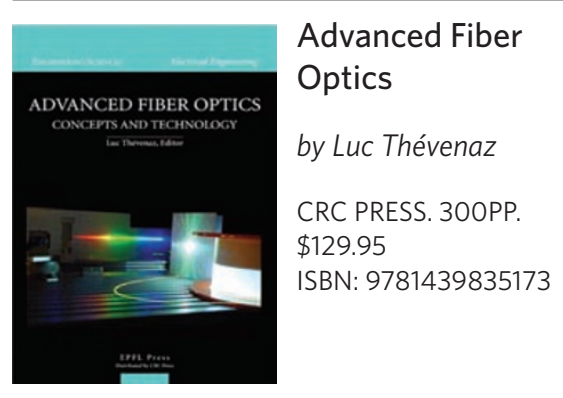

The aim of this book is to provide advanced students and young researchers with extensive knowledge about optical functions and their novel uses in the toolkit of advanced fibre optics. The first chapter summarizes the basic properties of optical fibres. The following chapters address the advanced features of fibre optics, such as light polarization, amplification, nonlinear optical interactions, photonic crystal fibres and supercontinuum generation. The book also covers elastic and inelastic scattering, along with their applications to distributed sensing.

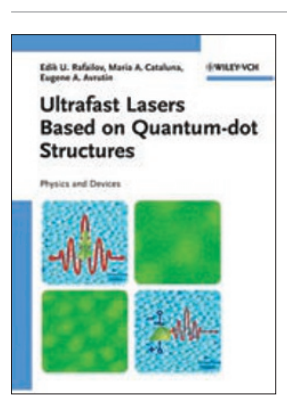

\section{Ultrafast Lasers Based on Quantum- dot Structures \\ by Edik U. Rafailov, Maria Ana Cataluna and Eugene A. Avrutin \\ WILEY. 262PP. $\$ 130.00$ ISBN: 9783527409280}

This volume addresses the physics, engineering and latest achievements of efficient and compact ultrafast lasers based on novel quantum-dot structures and devices. The approaches described by the authors encompass a broad range of laser systems while taking into consideration not only the physical and experimental aspects but also the required modelling tools, thus providing a holistic understanding of this popular topic.

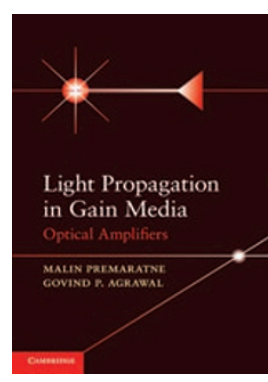

Light Propagation in Gain Media

by Malin Premaratne and Govind P. Agrawal

CAMBRIDGE UNIVERSITY PRESS. 284PP. $\$ 99.00$ ISBN: 9780521493482

This publication provides a comprehensive treatment of the fundamental concepts, theory and analytical techniques behind modern optical amplifier technology. It covers all the major optical amplification schemes for conventional materials, including the Raman and parametric gain processes. The final chapter is devoted to optical gain in metamaterials - a topic that has attracted considerable attention in recent years. The book emphasizes analytical insights to give a deeper, more intuitive understanding of various amplification schemes. It assumes background knowledge of electrical engineering or applied physics, including exposure to electrodynamics and wave motion, and is ideal for graduate students and researchers in physics, optics, biooptics and communications. 